\title{
Avaliação das práticas de uso de plantas medicinais no Município de Caxias-MA
}

\author{
Evaluation of practices for the use of medicinal plants in the City of Caxias-MA \\ Evaluación de prácticas para el uso de plantas medicinales en la Ciudad de Caxias-MA
}

Recebido: 12/03/2021 | Revisado: 17/03/2021 | Aceito: 20/03/2021 | Publicado: 01/04/2021

\author{
Antônia Fernanda Lopes da Silva \\ ORCID: https://orcid.org/0000-0003-1296-8767 \\ Universidade Estadual do Maranhão, Brasil \\ E-mail: nandalopesslp@hotmail.com \\ Luciano André Assunção Barros \\ ORCID: https://orcid.org/0000-0002-9927-4575 \\ Universidade Estadual do Maranhão, Brasil \\ E-mail: lucianoaab3@gmail.com
}

\begin{abstract}
Resumo
O objetivo deste trabalho foi avaliar as práticas de uso de plantas medicinais e suas principais formas de preparação por moradores de um bairro no município de Caxias-MA. Trata-se de uma pesquisa descritiva, com abordagem quantitativa, realizada em um bairro do município de Caxias-Ma. Para a coleta dos dados foi utilizado um questionário semiestruturado contendo as variáveis socioeconômicas e questões abertas e fechadas sobre o uso de plantas medicinais. Para verificar a associação entre as variáveis foi utilizado o teste Exato de Fisher, sendo os dados analisados através do programa SPSS. Contabilizou-se 97 entrevistados, destes, apenas 89,7\% referiram fazer o uso de plantas. Dentre estes, $86,2 \%$ eram do sexo feminino e 13,8\% eram do sexo masculino. A variável renda apresentou significância estatística (p-0,034), onde 55,2\% dos entrevistados afirmaram viver com menos de um salário-mínimo. Foi possivel identificar 22 plantas citadas pela população, sendo indicadas, principalmente, para má digestão, insônia, hipertensão, tosse, gripe e inflamação. Quando interrogados sobre com quem aprenderam sobre o uso de plantas, $66,0 \%$ relataram ter aprendido com ascendentes/familiares/avós/mães, enquanto nenhum dos entrevistados receberam informações oriundas de profissionais de saúde. Neste ponto, é importante que o profissional de saúde tenha conhecimento sobre as formas de uso e efeitos das plantas medicinais para que seja possível difundir mais informações sobre estas entre a população.
\end{abstract}

Palavras-chave: Plantas medicinais; Atenção primária à saúde; Educação em saúde.

\begin{abstract}
The objective of this work was to evaluate the practices of using medicinal plants and their main forms of preparation by residents of a neighborhood in the city of Caxias-MA. This is a descriptive research, with a quantitative approach, carried out in a neighborhood in the city of Caxias-MA. This is a descriptive research, with a quantitative approach, carried out in a neighborhood in the city of Caxias-MA. For data collection, a semi-structured questionnaire containing socioeconomic variables and open and closed questions about the use of medicinal plants was used. To verify the association between the variables, Fisher's exact test was used, and the data were analyzed using the SPSS program. 97 respondents were counted, of which only $89.7 \%$ reported using plants. Among these, $86.2 \%$ were female and $13.8 \%$ were male. The income variable showed statistical significance (p-0.034), where 55.2\% of respondents said they lived on less than one minimum wage. It was possible to identify 22 plants cited by the population, being mainly indicated for poor digestion, insomnia, hypertension, cough, flu and inflammation. When asked about who they learned about using plants, 66.0\% reported having learned from their ancestors / relatives / grandparents / mothers, while none of the interviewees received information from health professionals. At this point, it is important that the health professional has knowledge about the forms of use and effects of medicinal plants so that it is possible to disseminate more information about these among the population.
\end{abstract}

Keywords: Medicinal plants; Primary health care; Health education.

\section{Resumen}

El objetivo de este trabajo fue evaluar las prácticas de uso de plantas medicinales y sus principales formas de preparación por parte de vecinos de un barrio de la ciudad de Caxias-MA. Se trata de una investigación descriptiva, con enfoque cuantitativo, realizada en un barrio de la ciudad de Caxias-MA. Para la recolección de datos se utilizó un cuestionario semiestructurado que contiene variables socioeconómicas y preguntas abiertas y cerradas sobre el uso de plantas medicinales. Para verificar la asociación entre las variables se utilizó la prueba exacta de Fisher y los datos se analizaron mediante el programa SPSS. Se contabilizaron 97 encuestados, de estos, solo el 89,7\% informó usar plantas. Entre estos, el 86,2\% eran mujeres y el 13,8\% eran hombres. La variable ingreso mostró significancia estadística (p-0.034), donde el 55.2\% de los entrevistados dijo vivir con menos de un salário-mínimo. Se logró 
identificar 22 plantas citadas por la población, siendo indicadas, principalmente, para mala digestión, insomnio, hipertensión, tos, gripe e inflamación. Cuando se les preguntó sobre quiénes aprendieron sobre el uso de plantas, el 66.0\% informó haber aprendido de sus antepasados / familiares / abuelos / madres, mientras que ninguno de los entrevistados recibió información de profesionales de la salud. En este punto, es importante que el profesional de la salud tenga conocimiento sobre las formas de uso y efectos de las plantas medicinales para que sea posible difundir más información sobre estas entre la población.

Palabras clave: Plantas medicinales; Atención primaria de salud; Educación para la salud.

\section{Introdução}

O uso de plantas medicinais para alívio e cura de enfermidades é uma das primeiras práticas realizadas pelo ser humano (Zuanazzi \& Mayorga, 2010). Sendo considerada, no Brasil, uma prática comum que é reforçada pela diversidade cultural decorrente da colonização por populações europeias e africanas e pelo conhecimento tradicional indígena (Oliveira et al., 2011). Corroborando com os dados da Organização Mundial da Saúde (OMS), Oliveira et al., (2012) apontam que cerca de $80 \%$ da população mundial utiliza os princípios da medicina tradicional na atenção primária à saúde.

A adoção da Política Nacional de Práticas Integrativas e Complementares no Sistema Único de Saúde (SUS), a partir de 2006, permitiu um maior acesso ao conhecimento das plantas medicinais brasileiras e seu emprego na recuperação e manutenção da saúde. Alguns estados e municípios brasileiros vêm realizando nas duas últimas décadas a implantação de Programas de Fitoterapia na atenção primária à saúde com o intuito de suprir as carências medicamentosas de suas comunidades (Brasil, 2012).

Muitos dos programas de fitoterapia desenvolvidos no sistema público de saúde estão, atualmente, vinculados à Estratégia de Saúde da Família (ESF) uma vez que o uso de plantas medicinais e fitoterápicos é uma prática terapêutica alternativa e/ou complementar bastante utilizada pelas comunidades em geral (Teixeira et al., 2014).

O uso da fitoterapia na atenção primária é tecnicamente apropriado quando a prescrição é feita de forma racional e quando os profissionais não incentivam a medicalização excessiva. A atenção às possibilidades alternativas de tratamento levaria a uma melhora no atendimento da população pelo Sistema Único de Saúde, em razão de proporcionar outra forma de tratamento e de prevenção, principalmente em áreas onde a população possui baixo poder aquisitivo (Bruning, 2012).

Portanto, resgatar este conhecimento e suas técnicas terapêuticas é uma maneira de deixar registrado um modo de aprendizado informal que contribui para a valorização da medicina popular, além de gerar informações sobre a saúde da comunidade local. Diante deste contexto, o objetivo deste trabalho foi avaliar as práticas de uso de plantas medicinais e suas principais formas de preparação por moradores de um bairro no município de Caxias-MA.

\section{Metodologia}

Trata-se de uma pesquisa descritiva, com abordagem quantitativa, realizada no bairro Luiza Queiroz, município de Caxias-MA, no período de maio a setembro de 2020. Este bairro é um local originado de uma invasão, apresentando diversas vulnerabilidades sociais, sendo escolhido para realização da pesquisa por conter moradores oriundos da zona rural que, culturalmente, fazem o uso de plantas medicinais como alternativa para o tratamento de diversas patologias.

A população para o presente estudo foi composta por moradores de ambos os sexos. A população do bairro é assistida pela Estratégia de Saúde da Família (ESF) desde 2016, onde, atualmente, são cadastradas 430 famílias.

Para a coleta de dados foi utilizado um questionário semiestruturado, elaborado pelos autores, contendo as variáveis socioeconômicas e sociodemográficas, como estilo de vida, fatores clínicos e terapêuticos da população em estudo e questões abertas e fechadas referente ao tema abordado. A aplicação do questionário foi realizada nas dependências da Unidade de Saúde do bairro, quando o participante procurava por atendimento. Não sendo possível a coleta na residência em virtude da pandemia de Covid-19. 
Adotou-se como critérios de inclusão os seguintes dados: ser morador fixo do bairro há mais de dois anos, ser maior de 18 anos e aceitar participar da pesquisa de forma voluntária mediante a assinatura do Termo de Consentimento Livre e Esclarecido. E para os critérios de exclusão foram utilizados os seguintes: indivíduos que apresentam transtorno mental ou quadro clínico senil avançado ou diagnóstico de demência, confirmado em prontuário por profissionais da ESF.

Para caracterização da amostra foram analisadas as frequências absolutas (n) e relativas (\%). Para verificar associação entre as variáveis qualitativas foi usado o teste Exato de Fisher. Os dados foram tabulados em planilha eletrônica Microsoft Office Excel e analisados no programa IBM Statistical Package for the Social Sciences (SPSS) versão 20.0. O nível de significância adotado foi de $\mathrm{p} \leq 0,05$.

O trabalho foi aprovado pelo Comitê de Ética e Pesquisa (CEP) da Universidade Estadual do Maranhão sob o parecer n 4.002.121, com base nos princípios éticos da Resolução 466/2012 do Conselho Nacional de Saúde que trata de pesquisa envolvendo seres humanos.

\section{Resultados e Discussão}

Contabilizou-se um total de 97 entrevistados, destes 89,7\% ( $\mathrm{n}=87$ ) referiram fazer o uso de plantas medicinais. A caracterização socioeconômica, conforme descrito na Tabela 1, aponta que houve predominância do sexo feminino entre os entrevistados que fazem uso de plantas medicinais, sendo $86,2 \%$ ( $n=75$ ), enquanto o sexo masculino teve uma frequência de 13,8\% ( $n=12$ ). Este estudo corrobora com o estudo de Melo (2018), onde 73,6\% das entrevistadas eram do sexo feminino. Afirma ainda, que esta proporção pode ser justificada porque a mulher procura com mais frequência os serviços de saúde, seja para atendimento próprio ou como acompanhante dos filhos (Melo, 2018).

A idade dos participantes variou entre 18 a maiores de 55 anos sendo que a faixa etária de 36 a 45 anos foi a mais prevalente, com 25,3\% ( $n=22$ ), enquanto no estudo de Oliveira, Machado e Rodrigues (2014), a predominância de idade foi para maior de 59 anos com 47,46\% dos participantes. Em relação à escolaridade, observa-se que o ensino fundamental incompleto predominou, com 44,8\% (n=39), achado semelhante ao estudo de Löbler (2014), onde 38\% dos entrevistados não completaram o ensino fundamental. Sabe-se que o uso de plantas medicinais é frequente em todos os níveis de escolaridade, no entanto, neste estudo não foi possível estabelecer uma relação entre o nível de escolaridade e o uso de plantas.

Já em relação a renda, houve predominância de participantes que vivem com menos de um salário-mínimo, 55,2\% $(\mathrm{n}=48)$. Esta variável apresentou significância estatística $(\mathrm{p}=0,034)$, demonstrando que, quanto menor a renda, maior o uso de plantas medicinais. Löbler (2014), destaca que a baixa escolaridade está relacionada a baixa renda, como pode ser observado no presente estudo, tornando o uso de plantas medicinais uma alternativa mais barata e acessível à esta população, uma vez que o cultivo se faz nas próprias residências. Ressalta-se que as famílias deste bairro apresentam baixo poder aquisitivo, bem como o nível de analfabetismo elevado, no entanto o conhecimento sobre práticas populares é evidente. 
Tabela 1 - Variáveis sociodemográficas dos entrevistados sobre o uso de plantas medicinais dos moradores do bairro Luiza Queiroz do município de Caxias - MA. 2020.

\begin{tabular}{|c|c|c|c|c|c|}
\hline \multirow{3}{*}{ Variáveis } & \multicolumn{4}{|c|}{ Faz uso de plantas } & \multirow{3}{*}{ p-valor } \\
\hline & \multicolumn{2}{|l|}{ Não } & \multicolumn{2}{|l|}{ Sim } & \\
\hline & $\mathbf{n}=\mathbf{1 0}$ & $\%$ & $\mathrm{n}=\mathbf{8 7}$ & $\%$ & \\
\hline \multicolumn{6}{|l|}{ Sexo } \\
\hline Feminino & 8 & $(80,0)$ & 75 & $(86,2)$ & 0,634 \\
\hline Masculino & 2 & $(20,0)$ & 12 & $(13,8)$ & \\
\hline \multicolumn{6}{|l|}{ Idade } \\
\hline Entre 18 e 25 anos & 3 & $(30,0)$ & 10 & $(11,5)$ & 0,469 \\
\hline Entre 26 e 35 anos & 1 & $(10,0)$ & 15 & $(17,2)$ & \\
\hline Entre 36 e 45 anos & 2 & $(20,0)$ & 22 & $(25,3)$ & \\
\hline Entre 46 e 55 anos & 1 & $(10,0)$ & 21 & $(24,1)$ & \\
\hline Maior que 55 anos & 3 & $(30,0)$ & 19 & $(21,8)$ & \\
\hline \multicolumn{6}{|l|}{ Escolaridade } \\
\hline Analfabeto & 1 & $(10,0)$ & 17 & $(19,5)$ & 0,945 \\
\hline Ensino Fundamental Incompleto & 5 & $(50,0)$ & 39 & $(44,8)$ & \\
\hline Ensino Fundamental Completo & 1 & $(10,0)$ & 8 & $(9,2)$ & \\
\hline Ensino Médio Incompleto & 2 & $(20,0)$ & 11 & $(12,6)$ & \\
\hline Ensino Médio Completo & 1 & $(10,0)$ & 10 & $(11,5)$ & \\
\hline Ensino Super Incompleto & - & - & 1 & $(1,1)$ & \\
\hline Ensino Super Completo & - & - & 1 & $(1,1)$ & \\
\hline \multicolumn{6}{|l|}{ Etnia } \\
\hline Branco & 1 & $(10,0)$ & 3 & $(3,4)$ & 0,301 \\
\hline Pardo & 7 & $(70,0)$ & 53 & $(60,9)$ & \\
\hline Preto & 2 & $(20,0)$ & 31 & $(35,6)$ & \\
\hline \multicolumn{6}{|l|}{ Estado civil } \\
\hline Casado & 6 & $(60,0)$ & 62 & $(71,3)$ & 0,609 \\
\hline Solteiro & 4 & $(40,0)$ & 22 & $(25,3)$ & \\
\hline Viúvo & - & - & 3 & $(3,4)$ & \\
\hline \multicolumn{6}{|l|}{ Religião } \\
\hline Católica & 7 & $(70,0)$ & 51 & $(58,6)$ & 0,750 \\
\hline Evangélica & 1 & $(10,0)$ & 20 & $(23,0)$ & \\
\hline Nenhuma & 2 & $(20,0)$ & 15 & $(17,2)$ & \\
\hline Umbanda & - & - & 1 & $(1,1)$ & \\
\hline \multicolumn{6}{|l|}{ Renda } \\
\hline$<1$ salário & 9 & $(90,0)$ & 48 & $(55,2)$ & $\mathbf{0 , 0 3 4}$ \\
\hline > 1 salário & 1 & $(10,0)$ & 5 & $(5,7)$ & \\
\hline Igual 1 salário & - & - & 34 & $(39,1)$ & \\
\hline \multicolumn{6}{|l|}{ Ocupação } \\
\hline $\mathrm{ACS}$ & - & - & 1 & $(1,1)$ & 0,543 \\
\hline Autônomo & 1 & $(10,0)$ & 4 & $(4,6)$ & \\
\hline Auxiliar de Serviços & - & - & 3 & $(3,4)$ & \\
\hline Carpinteiro & - & - & 1 & $(1,1)$ & \\
\hline Comerciante & - & - & 3 & $(3,4)$ & \\
\hline Diarista & - & - & 3 & $(3,4)$ & \\
\hline Doméstica & 4 & $(40,0)$ & 34 & $(39,1)$ & \\
\hline Lavradora & 4 & $(40,0)$ & 36 & $(41,4)$ & \\
\hline Pescadora & - & - & 1 & $(1,1)$ & \\
\hline Soldador & 1 & $(10,0)$ & - & - & \\
\hline Vigilante & - & - & 1 & $(1,1)$ & \\
\hline
\end{tabular}


A ocupação dos entrevistados mostrou-se heterogênea, sendo lavrador 41,4\% ( $\mathrm{n}=36)$, seguido de doméstica 39,1\% $(\mathrm{n}=34)$ as ocupações que mais predominaram. Quanto à etnia, 60,9\% $(\mathrm{n}=53)$ dos entrevistados que fazem uso de plantas se autodeclararam pardos, seguido de 35,6\% ( $\mathrm{n}=31)$ negros e 3,4\% ( $\mathrm{n}=3)$ brancos. Quanto ao estado civil, 71,3\% (n=62) são casados, 25,3\% (n=22) são solteiros e 3,4\% (n=3) relataram serem viúvos.

Por meio dos entrevistados foi possível contabilizar 22 plantas para uso medicinal, cujo nome científico, nome popular, parte da planta utilizada e indicação de uso estão descritos na Tabela 2. Dentre estas, o Plectranthus barbatus (boldo), Melissa officinalis (cidreira), Cymbopogon citratus (capim santo), Mentha spicata (hortelã), Malva sylvestris (malva do reino) e Chenopodium ambrosioides (mastruz) foram as mais citadas, corroborando com o estudo de Madeiro e Lima (2017), onde Peumus boldus, Melissa officinalis e Cymbopogon citratus foram também as mais citadas.

No presente trabalho, o Plectranthus barbatus teve indicação de uso para má digestão e Covid-19. Na medicina popular, o uso desta planta é comum para problemas digestivos e hepáticos, tendo a boldina como principal alcalóide ativo responsável por essa ação. Apresenta ainda, atividade antioxidante, devido a presença de flavonóides (Ruiz, 2008). No entanto, até o momento da elaboração deste trabalho, não se encontrou dados na literatura científica acerca da indicação para uso em casos de Covid-19.

A Melissa officinalis foi a segunda planta mais citada, com indicação para insônia e hipertensão. Dentre os benefícios da M. officinalis, pode-se destacar o efeito calmante e sedativo, contribuindo para redução da insônia, ansiedade e estresse (Reis, 2009). Tornando-se uma das plantas medicinais mais utilizadas pela população para ansiedade, em virtude de suas características sensoriais que são bastante aceitas (Bortoluzzi, 2020). Além da M. officinalis, o Cymbopogon citratus (capim santo) também foi citado para insônia. Segundo Santos et al., (2009), C. citratus apresenta diversas atividades farmacológicas, voltadas para distúrbios menores, tais como ansiedade, insônia e má-digestão, comprovando seu uso pela população do presente estudo, assim como a M. officinalis.

É comum, entre pacientes com hipertensão, o uso de plantas medicinais com atividade hipotensora, como é o caso da M. officinalis e do C. citratus, que foram citados no estudo por terem efeitos vasorrelaxantes (Runnie et al., 2004). No entanto, os pacientes hipertensos devem ser orientados quanto ao uso destas plantas, uma vez que estas podem potencializar o efeito dos medicamentos anti-hipertensivos, contribuindo para o aparecimento de efeitos adversos, como a hipotensão (Kohlmann, 2010). 
Tabela 2 - Levantamento das principais plantas citadas por moradores do bairro Luiza Queiroz do município de Caxias - MA. 2020 .

\begin{tabular}{|c|c|c|c|c|c|}
\hline Planta & Nome Científico & $\begin{array}{c}\mathbf{N}^{\mathbf{0}} \\
\text { Citações }\end{array}$ & $\begin{array}{l}\text { Problema de } \\
\text { saúde/doença }\end{array}$ & Parte da Planta & Forma de uso \\
\hline Boldo & (Plectranthus barbatus) & 42 & Covid-19/má digestão & Folha & Chá \\
\hline Cidreira & (Melissa officinalis) & 35 & Insônia/Hipertensão & Folha & Chá \\
\hline Capim santo & (Cymbopogon citratus) & 19 & Insônia/Hipertensão & Folha & Chá \\
\hline Hortelã & (Mentha piperita) & 14 & Gripe & Folha & Chá \\
\hline Malva do reino & (Malva sylvestris) & 13 & Tosse/gripe & Folha & Lambedor \\
\hline Mastruz & $\begin{array}{l}\text { (Chenopodium } \\
\text { ambrosioides) }\end{array}$ & 10 & Inflamação & Folha & Emplastro \\
\hline Folha santa & (Kalanchoe brasiliensis) & 5 & Má digestão & Folha & Chá \\
\hline Limão & (Citrus limon) & 5 & Gripe & Casca & Chá \\
\hline Pau chapada & (Luetzelburgia auriculata) & 4 & Gastrite/azia & Casca do caule & Chá \\
\hline Dipirona & (Achillea millefolium) & 4 & Dores & Folha & Chá \\
\hline Insulina & (Cissus sicyoides) & 4 & Diabetes & Folha & Chá \\
\hline Tamarindo & (Tamarindus indica) & 3 & Hipertensão & Folha & Chá \\
\hline Inharé & (Brosimum gaudichaudii) & 3 & Micoses & Casca do caule & Chá \\
\hline Cravo & (Syzygium aromaticum) & 2 & Cefaleia & Semente & Chá \\
\hline Aroeira & (Schinus terebinthifolia) & 2 & Inflamação & Casca & Chá \\
\hline Jatobá & (Hymenaea courbaril) & 2 & Diabetes & Semente & Chá \\
\hline Algodão & (Gossypium hirsutum) & 2 & Inflamação & Folha & Chá \\
\hline Eucalipto & (Eucalyptus glubulus) & 2 & Febre & Folha & Chá \\
\hline Gengibre & (Zingiber officinale) & 2 & Dor de garganta & Rizoma & Chá \\
\hline Quebra pedra & (Phyllanthus niruri) & 1 & Cálculo renal & Folha & Chá \\
\hline Pata de vaca & (Bauhinia forficata) & 2 & Diabetes & Folha & Chá \\
\hline Alho & (Allium sativum) & 1 & Inflamação & "Dente" & Chá \\
\hline
\end{tabular}

Fonte: Autores (2020).

Dentre as plantas citadas para distúrbios respiratórios, como tosse e gripe, tem-se a Mentha piperita (hortelã), Malva sylvestris (malva do reino), Citrus limon (limão). A M. piperita apresenta atividades antiespasmódicas, estimulantes, carminativas e vermífuga (Grandi, 2014). Sendo bastante usada para infecções do trato respiratório (Ferreira, 2014). Estudos recentes estão sendo realizados para avaliar o potencial antimicrobiano do óleo essencial de M. piperita, dentre estes estudos cita-se o de Sujana et al., (2013), onde a presença do mentol, composto ativo do óleo essencial, foi associado à inibição de bactérias gram negativas e gram positivas, com potencial para tratamento de doenças infecciosas.

A M. sylvestris é conhecida por apresentar efeitos calmantes, expectorantes, anti-inflamatórios e antissépticos. Há estudos que comprovam o uso da planta para tosse e processo inflamatório das membranas mucosas, atribuindo esse efeito aos antioxidantes, taninos, óleos essenciais e flavonóides presente nas flores da planta (Cogo et al., 2010; Farina et al., 1995). No entanto, observa-se que a parte da planta citada para a forma a de uso é a folha. Diante disso, torna-se importante a orientação quanto às partes das plantas a serem utilizadas, uma vez que nem todas as partes apresentam uma concentração adequada de princípio ativo suficiente para o efeito terapêutico.

Outra planta citada pelos entrevistados foi a Chenopodium ambrosioides, conhecida popularmente como mastruz, sendo bastante utilizada para fins terapêuticos, principalmente na região nordeste do país (Silva, 2015). No presente estudo foi indicada por apresentar ação anti-inflamatória e cicatrizante, no entanto, sua ação mais comum e que a caracteriza como planta medicinal é a ação anti-helmíntica (Oliveira et al., 2018). Ainda assim, estudos recentes comprovam sua ação anti-inflamatória e cicatrizante, como o estudo de Souza et al., (2014), onde o extrato hidroalcóolico potencializou a atividade anti-inflamatória e antifibrótica em cirurgias de vesícula. O estudo de Sérvio et al., (2011) concluiu que o extrato aquoso da planta apresentou atividade cicatrizante em feridas abertas. 
Na Tabela 3 estão apresentadas informações sobre o uso das plantas medicinais pelos entrevistados. Quanto à frequência de uso das plantas, $46,4 \%$ ( $n=45)$ afirmam que fazem uso de forma frequente, enquanto $43,3 \%$ ( $n=42$ ) fazem uso raramente ou quando apresentam algum sintoma específico.

Quanto a forma de aquisição das plantas, 74,2\% $(\mathrm{n}=72)$ dos entrevistados relataram que adquirem nos quintais de suas casas, enquanto $11,3 \%(n=11)$ adquirem com os vizinhos e 4,1\% $(n=4)$ compram em feiras e mercados. Este estudo corrobora com Oliveira, Oliveira e Andrade (2010) onde 67,34\% dos entrevistados relataram adquirir as plantas de seus próprios quintais, enquanto lojas de produtos naturais e farmácias naturais representaram 10,02\% e 2, 04\% respectivamente.

Observou-se que o uso das plantas na forma de chá foi o mais citado pelos entrevistados, com uma frequência de 80,4\% ( $n=78)$, enquanto a forma de lambedor e emplastro, obtiveram uma frequência de 6,2\% ( $n=6)$ e 3,1\% ( $n=3$ ) respectivamente. Dados semelhantes foram encontrados no estudo de Filocreão (2017), onde a forma de chá (49,1\%) foi a mais citada pelos entrevistados. Os chás podem ser preparados por meio de infusão (adiciona-se a planta em água quente) ou decocção (adiciona-se a planta à água e em seguida leva-se ao fogo). A escolha do melhor método depende da parte da planta que será utilizada, levando em consideração a presença de óleos voláteis, sendo o método de infusão indicado para partes mais sensíveis da planta, como as folhas, flores e inflorescências, enquanto a decocção é indicada para partes mais duras, como as raízes, cascas e sementes (Vásquez, 2014).

Em relação ao aprendizado sobre as plantas e seus usos, $66 \%(n=64)$ dos entrevistados declararam ter aprendido com seus ascendentes/familiares/avós/mães, enquanto 22,7\% ( $\mathrm{n}=22)$ aprenderam com vizinhos. Estes são resultados comuns, uma vez que o conhecimento sobre as plantas medicinais é repassado de geração em geração. Destaca-se que o aprendizado repassado pelos profissionais de saúde não foi citado por nenhum dos entrevistados. Resultado semelhante foi encontrado no estudo de Silva (2015), onde 98,8\% dos entrevistados não receberam nenhuma orientação dos profissionais de saúde acerca do uso das plantas medicinais.

Ressalta-se que o uso das plantas medicinais ocorre frequentemente em associação ou substituição à terapia medicamentosa convencional, no entanto, o que é desconhecido pela população são os efeitos adversos/tóxicos que estas podem causar, uma vez que o uso de plantas não possui um controle rigoroso durante a prescrição e a venda, o que favorece à automedicação (Viganó, 2007; Macedo, 2007).

Em pacientes que possuem fatores de riscos evidentes, como problemas cardiológicos, a associação de plantas medicinais com medicamentos pode desencadear intoxicações severas ao organismo, levando pacientes à óbito (Teixeira et al., 2020). Diante disto, torna-se necessário que o profissional de saúde tenha conhecimento sobre a fitoterapia, difunda e oriente a população sobre o uso racional de plantas medicinais. 
Tabela 3 - Uso de plantas medicinais dos moradores do bairro Luiza Queiroz do município de Caxias - MA. 2020.

\begin{tabular}{|c|c|c|}
\hline Variáveis & $\mathrm{n}$ & $\%$ \\
\hline \multicolumn{3}{|l|}{ Faz uso de plantas } \\
\hline Não & 10 & 10,3 \\
\hline Sim & 87 & 89,7 \\
\hline \multicolumn{3}{|l|}{ Frequência de uso } \\
\hline Frequentemente & 45 & 46,4 \\
\hline Raramente & 42 & 43,3 \\
\hline Não se aplica/Não respondeu & 10 & 10,3 \\
\hline \multicolumn{3}{|l|}{ Formas de aquisição } \\
\hline Feiras/Mercados & 4 & 4,1 \\
\hline Quintal & 72 & 74,2 \\
\hline Vizinhos & 11 & 11,3 \\
\hline Não se aplica/Não respondeu & 10 & 10,3 \\
\hline \multicolumn{3}{|l|}{ Formas de uso } \\
\hline Chá & 78 & 80,4 \\
\hline Emplastro & 3 & 3,1 \\
\hline Lambedor & 6 & 6,2 \\
\hline Não se aplica/Não respondeu & 10 & 10,3 \\
\hline \multicolumn{3}{|l|}{ Aprendizado sobre as plantas } \\
\hline Ascendentes & 64 & 66,0 \\
\hline Vizinhos & 22 & 22,7 \\
\hline Profissional de saúde & 0 & 0,0 \\
\hline Não se aplica/Não respondeu & 11 & 11,3 \\
\hline \multicolumn{3}{|l|}{ Patologia } \\
\hline Hipertensão & 19 & 19,6 \\
\hline Diabetes/Hipertensão & 11 & 11,3 \\
\hline Artrite/Artrose & 3 & 3,1 \\
\hline Diabetes & 3 & 3,1 \\
\hline Dislipidemia & 2 & 2,1 \\
\hline Gastrite & 1 & 1,0 \\
\hline Hipertensão/Dislipidemia & 1 & 1,0 \\
\hline Não apresenta & 47 & 48,5 \\
\hline Não se aplica/Não respondeu & 10 & 10,3 \\
\hline
\end{tabular}

Fonte: Autores (2020).

Outro fator avaliado no estudo foi a presença ou ausência de patologia no indivíduo, sendo que 48,5\% (n=47) não apresentam patologia, 19,6\% $(\mathrm{n}=19)$ apresentaram hipertensão, 11,3\% $(\mathrm{n}=11)$ apresentaram hipertensão e diabetes, enquanto 3,1\% ( $n=3)$ tem diabetes. Ressalta-se que os entrevistados que afirmaram alguma patologia, tiveram confirmação do diagnóstico através da análise do prontuário.

Observa-se que o uso de plantas é uma prática comum entre a população estudada, sendo um importante recurso para a manutenção da saúde. No entanto, mais informações sobre o uso das plantas medicinais precisam ser difundidas entre a população, objetivando desmitificar o ditado popular “por ser natural, não faz mal”. Oliveira, Mezzomo e Moraes (2018), avaliaram o conhecimento de usuários de plantas em uma unidade de saúde, e quando interrogados sobre as restrições quanto ao uso, apenas $26,56 \%$ responderam que possuíam conhecimento sobre os efeitos adversos das plantas. 


\section{Considerações Finais}

Conclui-se que, no presente estudo, as plantas mais citadas foram Boldo (Plectranthus barbatus), Cidreira (Melissa officinalis), Capim santo (Cymbopogon citratus), Hortelã (Mentha piperita), Malva do reino (Malva sylvestris) e Mastruz (Chenopodium ambrosioides), sendo cultivados nos próprios quintais e utilizadas frequentemente na forma de chá. E o aprendizado sobre o uso dessas plantas medicinais é repassado de geração em geração, através dos familiares, avós e mães.

Diante dos resultados expostos é possível concluir que o uso de plantas medicinais é frequente entre a população estudada, sendo possível inferir, neste estudo, que a baixa renda é um fator que influencia no uso de plantas medicinais. É possível observar, ainda, que várias plantas citadas apresentam atividade biológica comprovada através de estudos recentes. No entanto, vale ressaltar que mais estudos científicos devem ser realizados para elucidar a ação de diversas plantas. Neste ponto, é importante que o profissional de saúde tenha conhecimento sobre as formas de uso e efeitos das plantas medicinais para que seja possível difundir mais informações sobre estas entre a população e assim, contribuir para o uso racional e seguro.

\section{Referências}

Brasil, Ministério da Saúde. (2012). Práticas Integrativas e Complementares. Plantas Medicinais e Fitoterapia na Atenção Básica. Normas e Manuais Técnicos-Caderno de Atenção Básica.

Bortoluzzi, M. M., Schmitt, V., \& Mazur, C. E. (2020). Efeito fitoterápico de plantas medicinais sobre a ansiedade: uma breve revisão. Research, Society and Development, 9(1), e02911504-e02911504. https://doi.org/10.33448/rsd-v9i1.1504.

Bruning, M. C. R., Mosegui, G. B. G., \& Vianna, C. M. D. M. (2012). A utilização da fitoterapia e de plantas medicinais em unidades básicas de saúde nos municípios de Cascavel e Foz do Iguaçu-Paraná: a visão dos profissionais de saúde. Ciência \& saúde coletiva, 17, $2675-2685$.

Cogo, L. L., Monteiro, C. L. B., Miguel, M. D., Miguel, O. G., Cunico, M. M., Ribeiro, M. L., \& Costa, L. M. D. (2010). Anti-Helicobacter pylori activity of plant extracts traditionally used for the treatment of gastrointestinal disorders. Brazilian Journal of Microbiology, 41(2), 304-309. https://doi.org/10.1590/S1517-83822010000200007.

Farina, A., Doldo, A., Cotichini, V., Rajevic, M., Quaglia, M. G., Mulinacci, N., \& Vincieri, F. F. (1995). HPTLC and reflectance mode densitometry of anthocyanins in Malva silvestris L.: a comparison with gradient-elution reversed-phase HPLC. Journal of pharmaceutical and biomedical analysis, 14(1-2), 203-211. https://doi.org/10.1016/0731-7085(95)01632-5.

Ferreira, A. R. A. (2014). Uso de óleos essenciais como agentes terapêuticos (Doctoral dissertation, [sn])

Filocreão, A. S. M., Galindo, A. G., \& dos Santos, T. D. J. S. (2017). Fitoterapia na Amazônia: a experiência do estado do Amapá-Brasil. Desenvolvimento e Meio Ambiente, 40. http://dx.doi.org/10.5380/dma.v40i0.43655.

Grandi, T. S. M. (2014). Tratado das plantas medicinais: mineiras, nativas e cultivadas: Adequatio Estúdio.

Kohlmann Jr, O., Gus, M., Ribeiro, A. B., Vianna, D., Coelho, E. B., Barbosa, E., \& Santos, R. A. S. D. (2010). Tratamento medicamentoso. Brazilian Journal of Nephrology, 32, 29-43.

Löbler, L., Santos, D., dos Santos Rodrigues, E., \& dos Santos, N. R. Z. (2014). Levantamento etnobotânico de plantas medicinais no bairro Três de Outubro, da cidade de São Gabriel, RS, Brasil. Revista Brasileira de Biociências, 12(2), 81.

Macedo, A. F., Oshiiwa, M., \& Guarido, C. F. (2007). Ocorrência do uso de plantas medicinais por moradores de um bairro do município de Marília-SP. Revista de Ciências Farmacêuticas Básica e Aplicada, 28(1).

Madeiro, A. A. S., \& de Lima, C. R. (2017). Levantamento etnofarmacológico das plantas medicinais utilizadas pelos usuários da USF Paulo Leal de Melo em Maceió-AL. Interfaces Científicas-Saúde e Ambiente, 5(2), 41-52. https://doi.org/10.17564/2316-3798.2017v5n2p41-52.

Melo, F. B. A. D. (2018). Uso de plantas medicinais e sua relação com a adesão ao tratamento medicamentoso entre pacientes portadores de diabetes no âmbito da atenção primária a saúde. Repositório da Universidade Federal de Alagoas-UFAL.

Oliveira, V. B. D., Mezzomo, T. R., \& Moraes, E. F. D. (2018). Conhecimento e uso de plantas medicinais por usuários de unidades básicas de saúde na região de Colombo, PR. Revista Brasileira de Ciências da Saúde, 22(1), 57-64. https://doi.org/10.4034/RBCS.2018.22.01.08.

Oliveira, R. A., Silva, R. V., Neves, V. L. D., de Oliveira Nascimento, I., dos Santos Oliveira, F., Nunes, S. E. A., \& Belfort, M. G. S. (2018). Perfil etnobotânico de plantas utilizadas como medicinais na comunidade de Bom Jesus, município de Imperatriz-MA. Educação Ambiental em Ação, 17(66).

Oliveira, L. A. R., Machado, R. D., \& Rodrigues, A. J. L. (2014). Levantamento sobre o uso de plantas medicinais com a terapêutica anticâncer por pacientes da Unidade Oncológica de Anápolis. Revista brasileira de plantas medicinais, 16(1), 32-40. http://dx.doi.org/10.1590/S1516-05722014000100005.

Oliveira, S. G. D, de Moura, F. R. R., Demarco, F. F., da Silva Nascente, P., Del Pino, F. A. B., \& Lund, R. G. (2012). Inquérito etnomedicinal sobre fitoterapia com profissionais e pacientes de Unidades Básicas de Saúde do Sistema Único de Saúde. Journal of Ethnopharmacology, 140 (2), $428-437$. 
Oliveira, A. K. M., Oliveira, N. A., Resende, U. M., \& Martins, P. F. R. B. (2011). Ethnobotany and traditional medicine of the inhabitants of the Pantanal Negro sub-region and the raizeiros of Miranda and Aquidauna, Mato Grosso do Sul, Brazil. Brazilian Journal of Biology, 71(1), 283-289. http://dx.doi.org/10.1590/S1519-69842011000200007.

Oliveira, G. L. de., Oliveira, A. F. M. de., \& Andrade, L. de H. C. Plantas medicinais utilizadas na comunidade urbana de Muribeca, Nordeste do Brasil. Acta Botanica Brasilica, 24(2), 571-577, 2010. http://dx.doi.org/10.1590/S0102-33062010000200026.

Reis, É. S., Pinto, J. E. B. P., Rosado, L. D. S., \& Corrêa, R. M. (2009). Teor e composição química do óleo essencial de Melissa officinalis L. in vitro sob influência do meio de cultura. Acta Scientiarum. Agronomy, 31(2), 331-335. http://dx.doi.org/10.1590/S1807-86212009000200021.

Runnie, I., Salleh, M. N., Mohamed, S., Head, R. J., \& Abeywardena, M. Y. (2004). Vasorelaxation induced by common edible tropical plant extracts in isolated rat aorta and mesenteric vascular bed. Journal of Ethnopharmacology, 92(2-3), 311-316. https://doi.org/10.1016/j.jep.2004.03.019.

Ruiz, A. L. T., Taffarello, D., Souza, V. H., \& Carvalho, J. E. (2008). Farmacologia e toxicologia de Peumus boldus e Baccharis genistelloides. Revista Brasileira de Farmacognosia, 18(2), 295-300. http://dx.doi.org/10.1590/S0102-695X2008000200025.

Santos, A., Paduan, R. H., Gazin, Z. C., Jacomassi, E., D'Oliveira, P. S., Cortez, D. A. G., \& Cortez, L. E. R. (2009). Determinação do rendimento e atividade antimicrobiana do óleo essencial de Cymbopogon citratus (DC.) Stapf em função de sazonalidade e consorciamento. Revista Brasileira de Farmacognosia, 19(2A), 436-441. http://dx.doi.org/10.1590/S0102-695X2009000300017.

Sérvio, E. M. L., de Araújo, K. S., da Silva Nascimento, L. R., da Costa, C. L. S., Mendes, L. M. S., Maia Filho, A. L. M., \& Santos, Í. M. S. P. (2011). Cicatrização de feridas com a utilização do extrato de Chenopodium ambrosioides (mastruz) e cobertura secundária estéril de gaze em ratos. ConScientiae Saúde, 10(3), 441-448. https://doi.org/10.5585/conssaude.v10i3.2664.

Silva, A. B., Araújo, C., \& Mariz, S. (2015). The use of medicinal plants for elderly users of a basic family health unit. J. Nurs. UFPE, Recife, 9(3).

Souza, J. P. J. D. (2014). Efeito anti-inflamatório do extrato hidroalcoólico de folhas de Chenopodium ambrosioides L. na bexiga de ratos submetidos à cistotomia. Repositório da Universidade Federal do Maranhão-UFMA.

Sujana, P., Sridhar, T. M., Josthna, P., \& Naidu, C. V. (2013). Antibacterial activity and phytochemical analysis of Mentha piperita L. (Peppermint)—An important multipurpose medicinal plant. American Journal of Plant Sciences 4 (1), 27633, 7. https://doi.org/10.4236 / ajps.2013.41012.

Teixeira, A. H., Bezerra, M. M., Chaves, H. V., do Val, D. R., Pereira Filho, S. M., \& Rodrigues, A. A. (2014). Conhecimento popular sobre o uso de plantas medicinais no município de Sobral-Ceará, Brasil. SANARE-Revista de Políticas Públicas, 13(1).

Silva Teixeira, J. P., Macedo, A. P. V., da Silva Cândido, G., Magalhães, J. K. A., da Silva, M. W., Nunes, H. M. L., \& da Silva, G. C. (2020). Perfil epidemiológico dos casos de intoxicação por plantas medicinais no Brasil de 2012 a 2016. Brazilian Journal of Development, 6(10), 82199-82209.

Vásquez, S. P. F., Mendonça, M. S. D., \& Noda, S. D. N. (2014). Etnobotânica de plantas medicinais em comunidades ribeirinhas do Município de Manacapuru, Amazonas, Brasil. Acta Amazonica, 44(4), 457-472. https://doi.org/10.1590/1809-4392201400423.

Viganó, J., Viganó, J. A., \& da Cruz Silva, C. T. A. (2007). Utilização de plantas medicinais pela população da região urbana de Três Barras do Paraná. Acta Scientiarum. Health Sciences, 29(1), 51-58.

Zuanazzi, J. A. S., \& Mayorga, P. (2010). Fitoprodutos e desenvolvimento econômico. Química nova, 33(6), 1421-1428. 